\title{
Adaptive Beam Formation in Smart Antenna Using VSLMS and VSNLMS Algorithms for Side Lobe Level Reduction
}

\author{
Monisha Bairagya \\ School of Electronics Engineering, KIIT University \\ Bhubaneswar 751024 (Odisha, India) \\ Email: monishabairagya92 [AT] gmail.com
}

\begin{abstract}
In mobile communication using smart antenna, the capacity of a cellular network can be enhanced significantly. Adaptive beamforming is one of the critical issues in smart antenna. A comparative study of beamforming algorithms by using their variable step size parameter like, variable step size least mean square algorithm (VSLMS) and variable step size normalized least mean square algorithm (VSNLMS) are presented in this paper. Smart antennas of 8 elements and 10 elements linear array are considered here. It is found that using VSNLMS algorithm a lower side lobe level is achieved compared to VSLMS algorithm for various angles of desired user and undesired interferer.
\end{abstract}

Keywords - Smart antenna, adaptive beamforming, VSLMS algorithm, VSNLMS algorithm, Side lobe reduction

\section{INTRODUCTION}

The overall performance in mobile communication can be improved by using the adaptive smart antenna which is an antenna array of any antenna with a smart signal processing unit. Smart antenna estimates the direction of arrival (DOA) of a signal, and generates radiation beam along the desired user and produces null towards the undesired interferer [1-2] (Figure 1). Appreciable power saving is possible using the smart antenna in the mobile network in addition to the enhancement of signal quality, network capacity, and coverage area. The adaptation is achieved by multiplying the incoming signal with complex weights and then summing them together to obtain the desired radiation pattern. Smart antenna generates side lobes which may produce interference for other users [1]. There are two basic types of smart antennas, viz., switched beam smart antenna and adaptive smart antenna. Switched beam systems have several available fixed beam patterns. A decision is made as to which beam to access, at any given point of time, based upon the requirements of the system. Adaptive arrays allow the antenna to steer the beam to any direction of interest while simultaneously nullifying interfering signals.

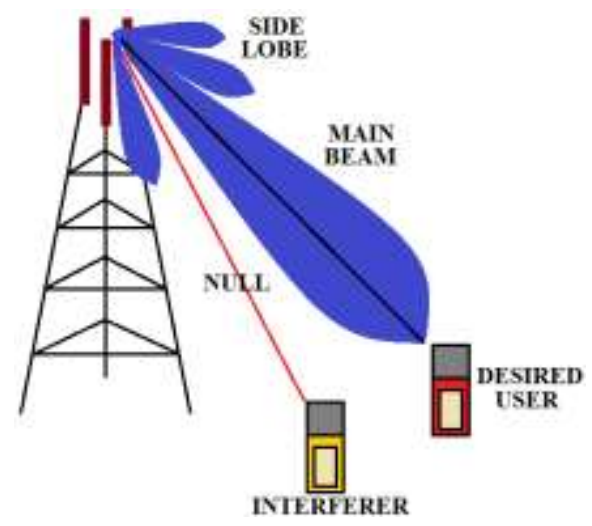

Figure 1: Main beam toward desired user and null toward interferer

To generate a beam in the desired direction and null toward the interferer various signal processing algorithms are used. Most popular algorithms for beamforming are least mean square (LMS) algorithm, recursive least square (RLS) algorithm, sample matrix inversion algorithm (SMI) [2-4]. The algorithms are used mainly for the smart antenna using 
linear antenna arrays. In addition to above algorithms, some combined and hybrid algorithms are also used for beamforming [5-7]. A better performance for reduction of side lobe level has achieved by using VSLMS algorithm [8].

In this paper, variable step-size least mean square (VSLMS) algorithm along with the variable step-size normalized least mean square (VSNLMS) algorithm are analyzed intensively for generation of an adaptive radiation beam in the smart antenna. Smart antenna produces side lobe which causes interference to other users. Attention is paid for reduction of the maximum side lobe level as much as possible. These algorithms are applied for beamforming in smart antenna for various values of the step-size parameter. For all the algorithms the effect of the step-size parameter on beamforming is studied and the best results are presented. Performances of those algorithms are studied for the smart antenna array of 8element \& 10-element isotropic elements and the element spacing is considered as $0.5 \lambda$. The performance of VSNLMS algorithm is found better than VSLMS algorithm for reduction of side lobe level.

\section{BEAMFORMING ALGORITHMS}

Least mean square (LMS) algorithm is a stochastic and a steepest descent method, where iterative procedure is used for making successive corrections to the weight vector in the direction of the negative of the gradient vector which eventually leads to the minimum mean square error [9]. Adaptive algorithm is used to minimize the error $e(n)$ between desired signal $d(n)$ and array output $y(n)[7,9]$.

$$
e(n)=d(n)-y(n)
$$

LMS algorithm updates the weight vectors according to the equation [9, 11].

$$
w(n+1)=w(n)+\mu x(n) e^{*}(n)
$$

Where $\mu$ is the step size parameter and $e^{*}(n)$ is the complex conjugate of $e(n)$. The step size $\mu$ should meet the condition

$$
0<\mu<\frac{1}{\lambda_{\max }}
$$

Where $\lambda_{\max }$ is the largest Eigen value of the array correlation matrix R.

Normalized least mean square (NLMS) is used to achieve faster convergence and stability of the algorithm. Here, the step-size is divided by the norm of the input signal to avoid gradient noise amplification due to $x(n)$. For normalized least mean square (NLMS) algorithm, the weight vector adaptation equation is [9, 11].

$$
w(n+1)=w(n)+\frac{\mu}{\|x(n)\|^{2}} x(n) e^{*}(n)
$$

Where the step size $\mu(n)$ is defined as

$$
\mu(n)=\frac{\mu}{\|x(n)\|^{2}}
$$

In VSLMS and VSNLMS algorithms the forward predicted weight vector and present weight vector are used to calculate a new parameter $\epsilon_{n}$ using the difference between the norms of both weight vectors as [10].

$$
\epsilon_{\mathrm{n}}=\frac{\|w(n+1)-w(n)\|}{\|w(n+1)\|}
$$

The $\epsilon_{n}$ is then passed to an alpha filter to generate the new convergence parameter $\mu_{n+1}$ that will be used to predict the weight vector $w(n+1)$.

$$
\mu_{n+1}=\left\{\begin{array}{cc}
\alpha \mu_{n}+\delta \epsilon_{n} & , 0<\mu_{n+1}<\mu_{\max } \\
\mu_{\max } & , \text { otherwise }
\end{array}\right.
$$

Where $\mu_{\max }$ is the maximum convergence parameter that can be used for LMS algorithm where $\mu_{\max }$ is defined as

$$
\mu_{\max }<\frac{2}{\lambda_{\max }}
$$

VSLMS algorithm updates the weight vectors according to the equation 


$$
w(n+1)=w(n)+\mu_{n+1} x(n) e^{*}(n)
$$

In variable step-size normalized least mean square (VSNLMS) algorithm, the step size $\mu$ is also being changed like VSLMS algorithm by following the above equations (6), (7) \& (8). So, the equation of weight updated VSNLMS algorithm is

$$
w(n+1)=w(n)+\frac{\mu_{n+1}}{\|x(n)\|^{2}} x(n) e^{*}(n)
$$

\section{BEAMFORMING IN SMART ANTENNA}

Figure 2 shows a uniform linear antenna array of isotropic elements with inter-element spacing of $d$. Antennas are excited by sinusoidal currents with progressive phase shift of $\alpha$.

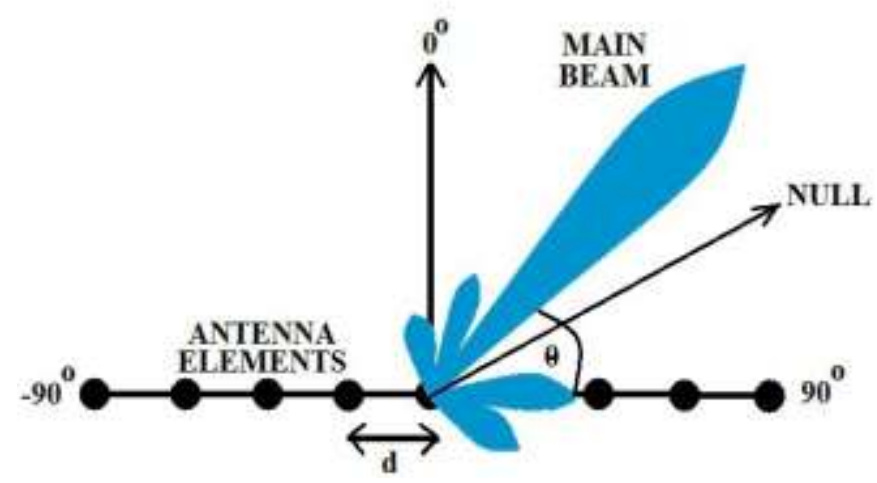

Figure 2: Linear antenna array configuration

Adaptive beam formation is done for a linear array of uniform element spacing of $0.5 \lambda$. For a main beam at an angle $\theta$, the array factor for $\mathrm{N}$ element linear array is given by [12]

$$
A F_{L}(\theta)=\sum_{n=1}^{N} A_{n} \times e^{j(n-1)}\left(\frac{2 \pi d}{\lambda} \cos \theta+\alpha\right)
$$

To generate main beam at wavelength $\lambda$, toward the desired beam direction $\theta^{0}$ from the broadside direction, the progressive phase shift is given by

$$
\alpha=\frac{-2 \pi d}{\lambda} \cos \theta_{0}
$$

Normalized array factor is

$$
A F_{\text {norm }}=\frac{A F}{A F_{\max }}
$$

Where, $A F_{\max }$ is the maximum value of array factor. 
In computer programming, there are two sets of values are considered for directions of main beam directions and null. Firstly it is assumed that the desired signal direction is at $15^{\circ}$ and the interferer is present at $0^{\circ}$. Secondly, it is assumed that the desired signal direction is at $30^{\circ}$ and the interferer is present at $60^{\circ}$. The number of iterations is 200 for each algorithm. The value of inter-element spacing is at $0.5 \lambda$. The adaptation equations are used to update the weights in VSLMS and VSNLMS algorithms respectively. The values of $\alpha$ and $\delta$ in equation (7) is taken as 0.95 and 0.003 respectively.

The normalized array factors obtained by using VSLMS and VSNLMS algorithms for antenna element of 8 and main beam direction at $15^{\circ}$ and null at $0^{\circ}$ and for step-size $(\mu)$ values $0.04,0.03,0.02 \& 0.01$ respectively are plotted in figure 3 , figure 4 , figure 5 and figure 6 respectively.

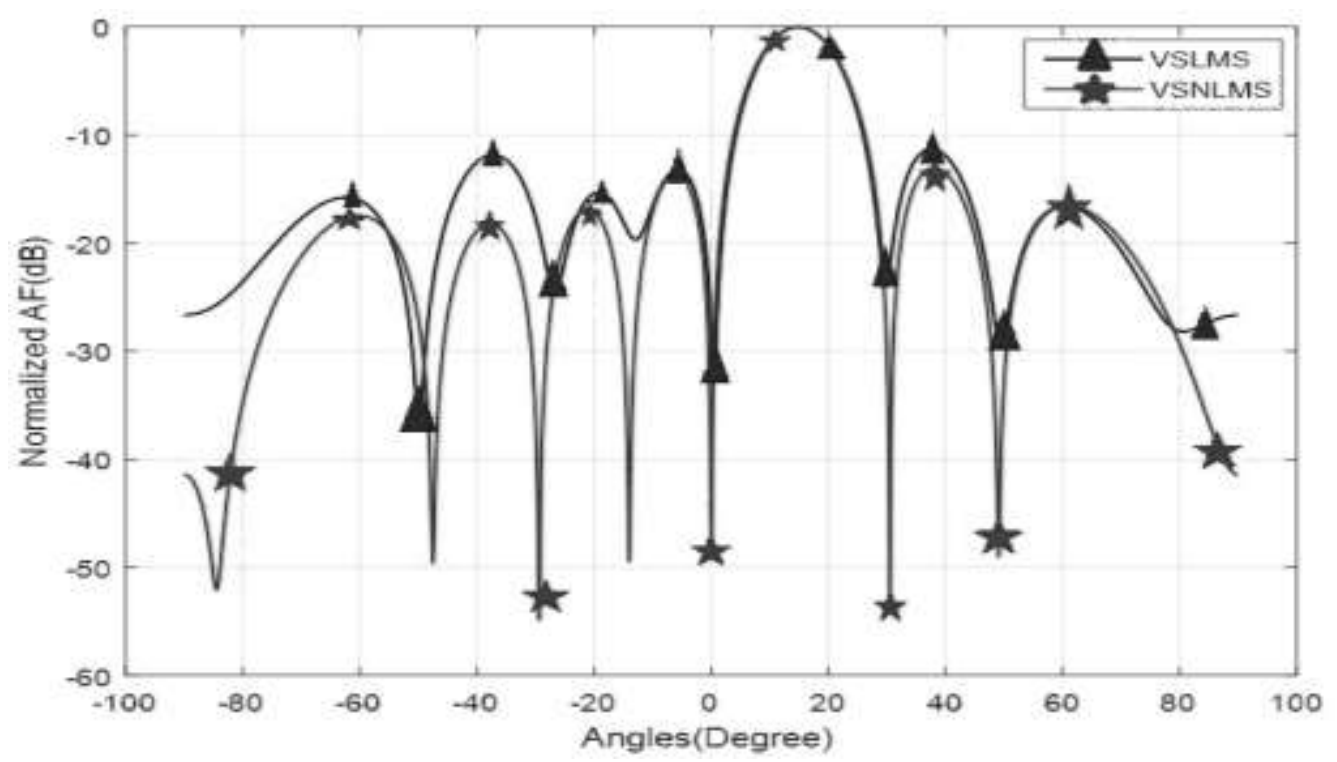

Figure 3: Normalized Array Factor for Antenna Element $N=8$ at Beam Direction $=15^{\circ}$, Null Direction $=0^{\circ}$ and $\mu=0.04$

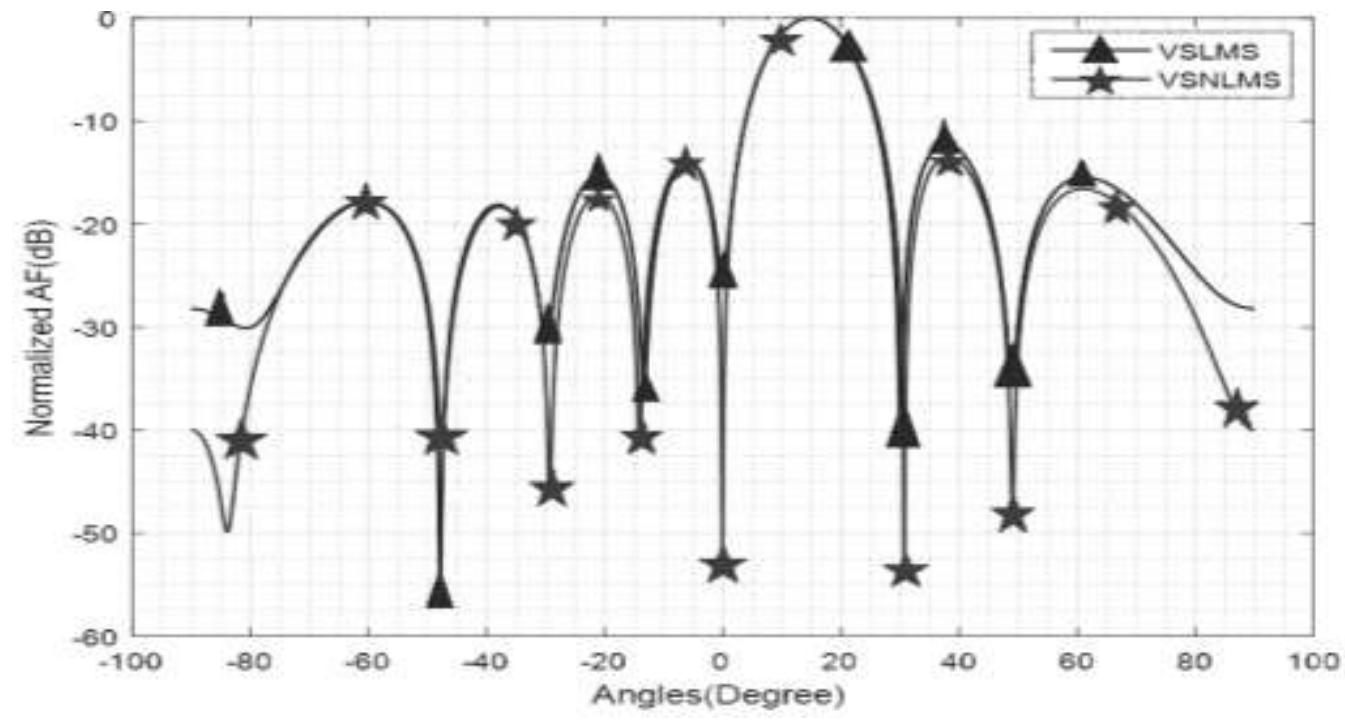

Figure 4: Normalized Array Factor for Antenna Element $\mathrm{N}=8$ at Beam Direction $=15^{\circ}$, Null Direction $=0^{\circ}$ and $\mu=0.03$ 


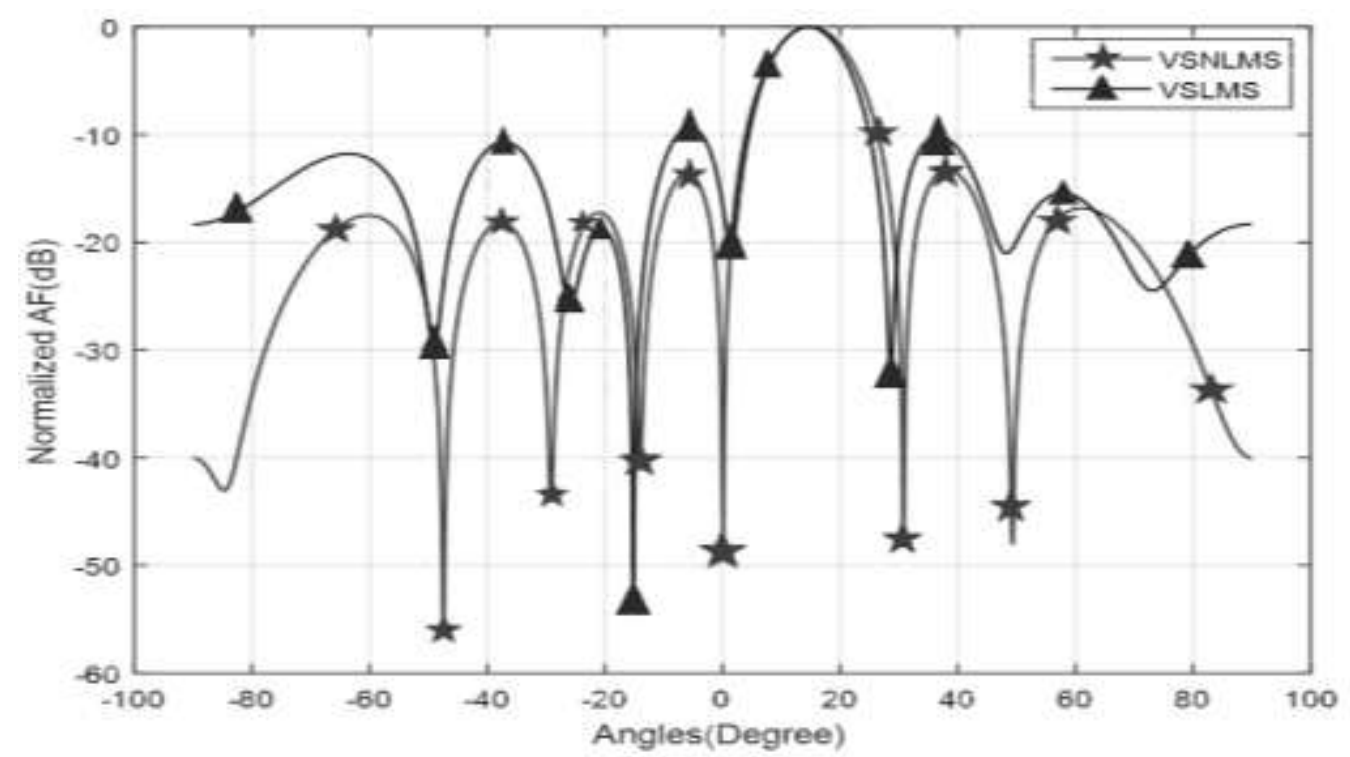

Figure 5: Normalized Array Factor for Antenna Element $N=8$ at Beam Direction $=15^{\circ}$, Null Direction $=0^{\circ}$ and $\mu=0.02$

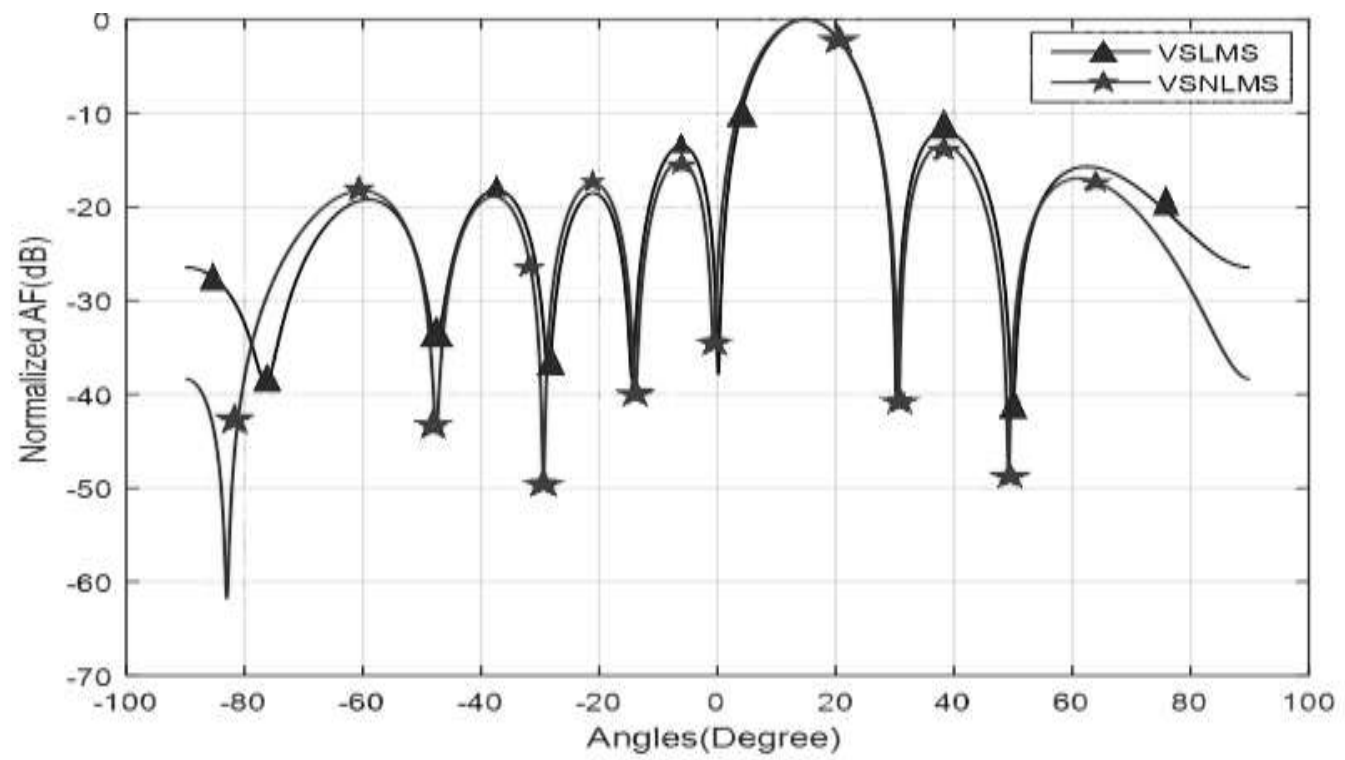

Figure 6: Normalized Array Factor for Antenna Element $\mathrm{N}=8$ at Beam Direction $=15^{\circ}$, Null Direction $=0^{\circ}$ and $\mu=0.01$

Table 1: Performances of Beamforming Algorithms for $\mathrm{N}=8$ at Beam Direction $=15^{\circ}$, Null Direction $=0^{\circ}$

\begin{tabular}{|c|c|c|c|c|c|}
\hline \multirow{2}{*}{$\boldsymbol{\mu}$} & Algorithms & $\begin{array}{c}\text { Simulated beam } \\
\text { direction (Deg) }\end{array}$ & $\begin{array}{c}\text { Null depth at desired } \\
\text { null direction (dB) }\end{array}$ & $\begin{array}{c}\text { Beamwidth } \\
(\mathbf{D e g})\end{array}$ & $\begin{array}{c}\text { SLL }_{\text {max }} \\
(\mathbf{d B})\end{array}$ \\
\hline \multirow{2}{*}{0.04} & VSLMS & $15.0^{\circ}$ & -32.5 & $28.4^{\circ}$ & -11.3 \\
\cline { 2 - 6 } & VSNLMS & $15.0^{\circ}$ & -48.5 & $30.0^{\circ}$ & -13.1 \\
\hline \multirow{2}{*}{0.03} & VSLMS & $15.0^{\circ}$ & -25.0 & $30.0^{\circ}$ & -12.2 \\
\cline { 2 - 6 } & VSNLMS & $15.0^{\circ}$ & -52.5 & $30.3^{\circ}$ & -13.5 \\
\hline \multirow{2}{*}{0.02} & VSLMS & $15.0^{\circ}$ & -22.3 & $28.2^{\circ}$ & -10.0 \\
\cline { 2 - 6 } & VSNLMS & $15.0^{\circ}$ & -47.5 & $30.2^{\circ}$ & -13.3 \\
\hline \multirow{2}{*}{0.01} & VSLMS & $15.0^{\circ}$ & -37.5 & $30.0^{\circ}$ & -11.9 \\
\cline { 2 - 6 } & VSNLMS & $15.0^{\circ}$ & -34.7 & $30.6^{\circ}$ & -13.4 \\
\hline
\end{tabular}


From Table 1, it is observed that null depth at desired null direction for VSNLMS algorithm is deeper than VSLMS algorithm. The normalized array factors obtained by using VSLMS and VSNLMS algorithms for antenna element of 10 and main beam direction at $30^{\circ}$, null at $60^{\circ}$ and for step-size $(\mu)$ values $0.04,0.03,0.02 \& 0.01$ respectively are plotted in figure 7 , figure 8 , figure 9 and figure 10 respectively.

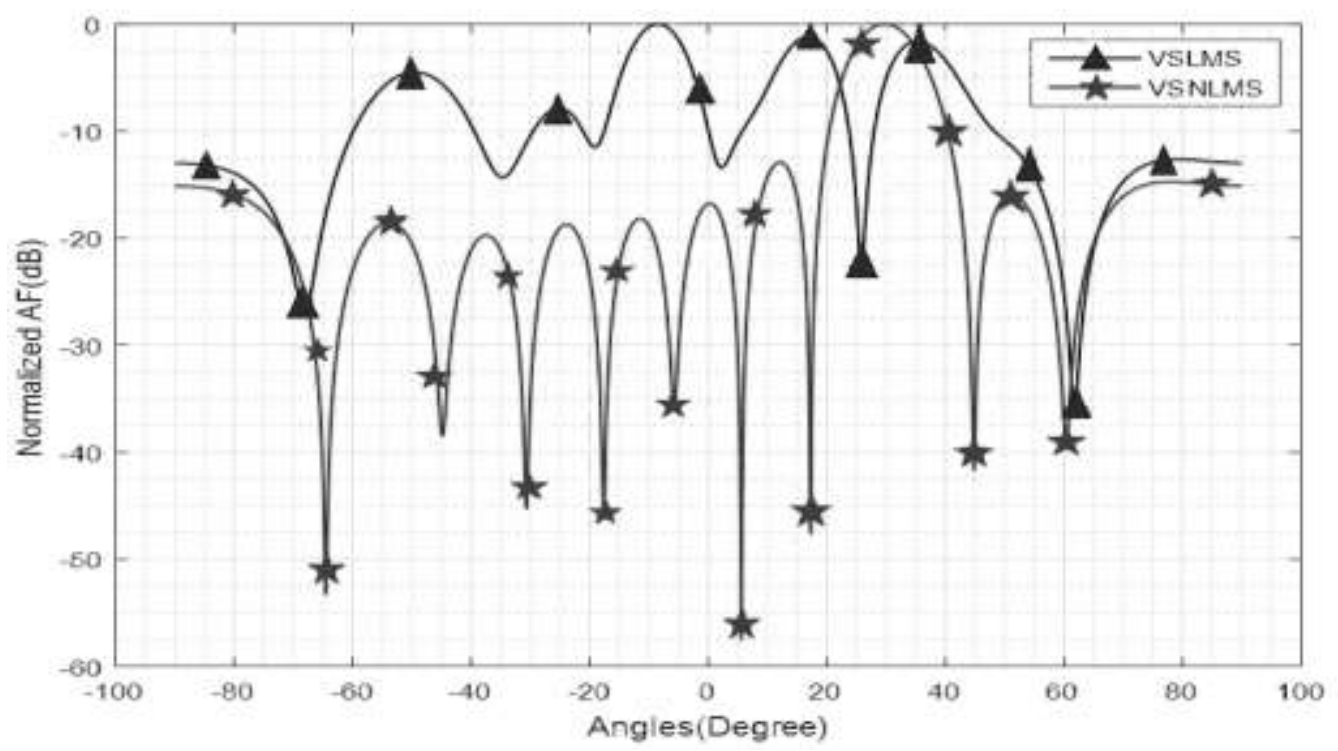

Figure 7: Normalized Array Factor for Antenna Element $\mathrm{N}=10$ at Beam Direction $=30^{\circ}$, Null Direction $=60^{\circ}$ and $\mu=0.04$

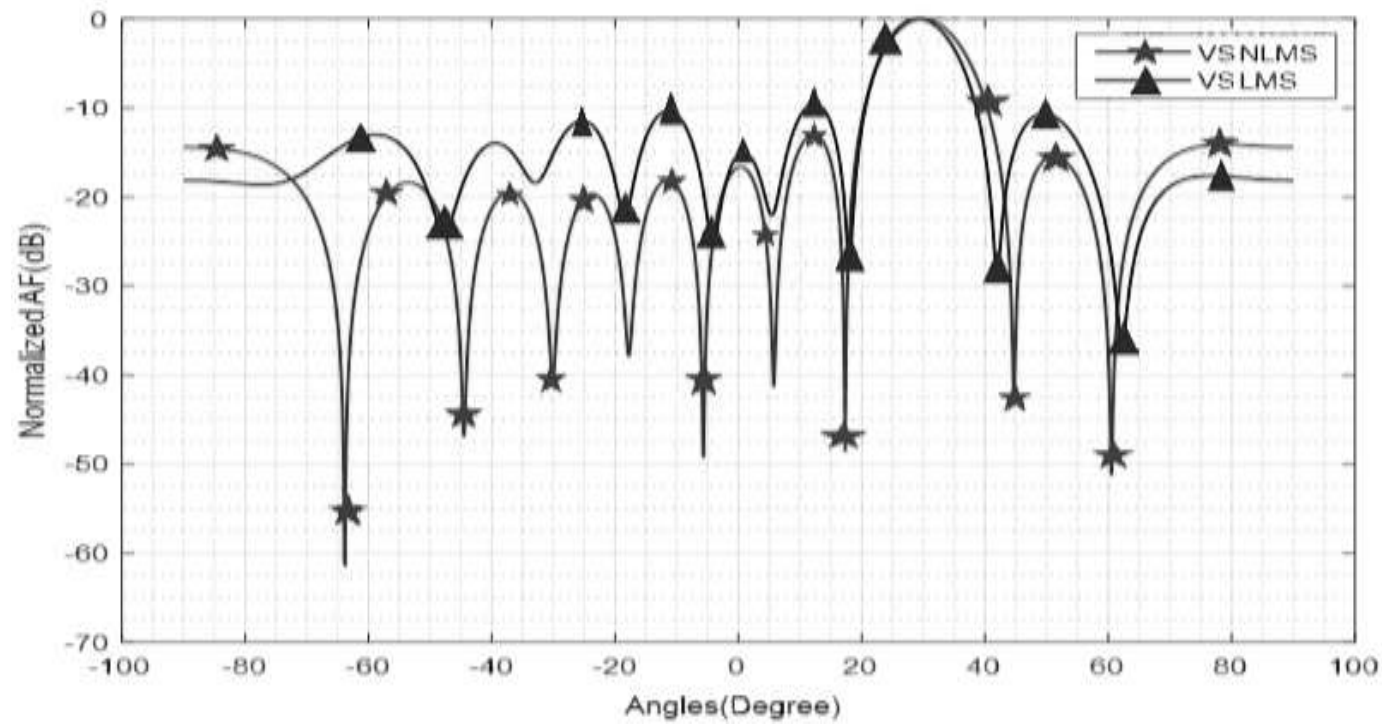

Figure 8: Normalized Array Factor for Antenna Element $\mathrm{N}=10$ at Beam Direction $=30^{\circ}$, Null Direction $=60^{\circ}$ and $\mu=0.03$ 


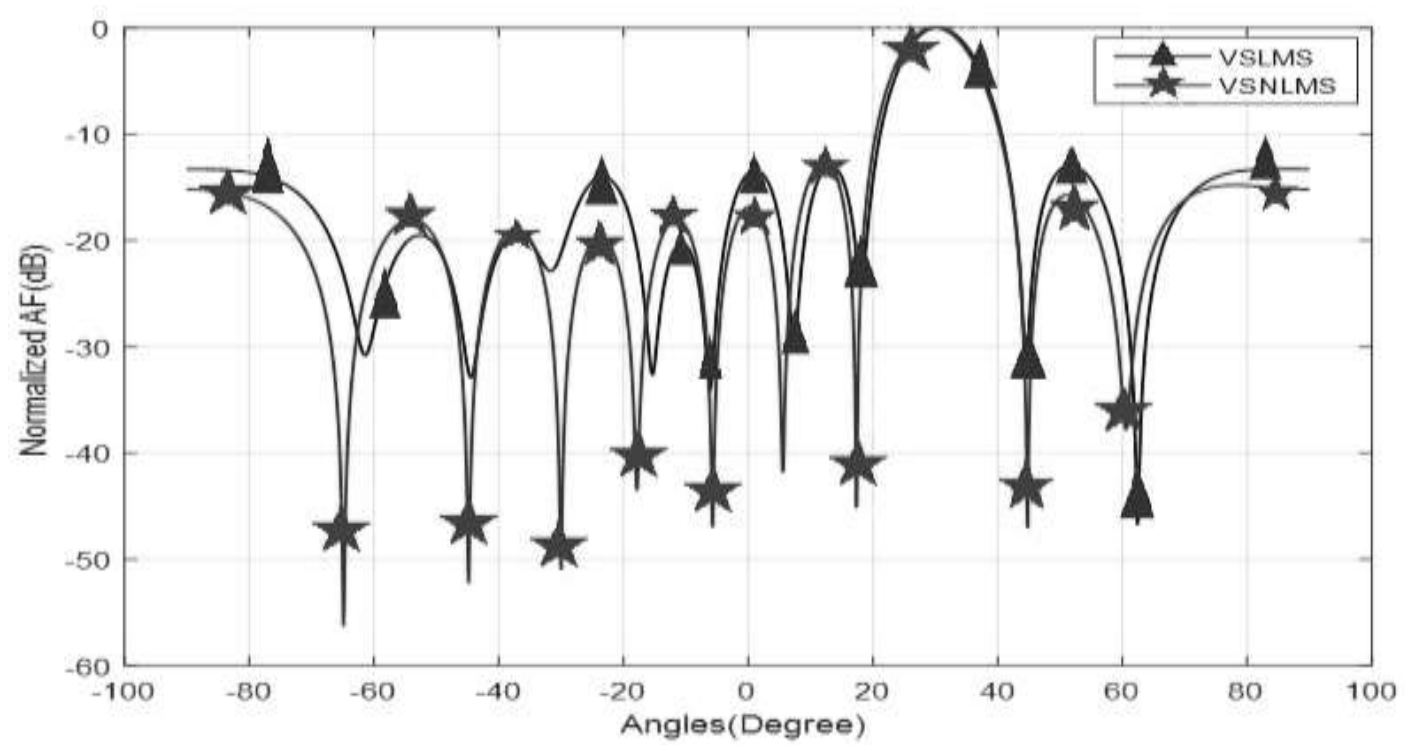

Figure 9: Normalized Array Factor for Antenna Element $\mathrm{N}=10$ at Beam Direction $=30^{\circ}$, Null Direction $=60^{\circ}$ and $\mu=0.02$

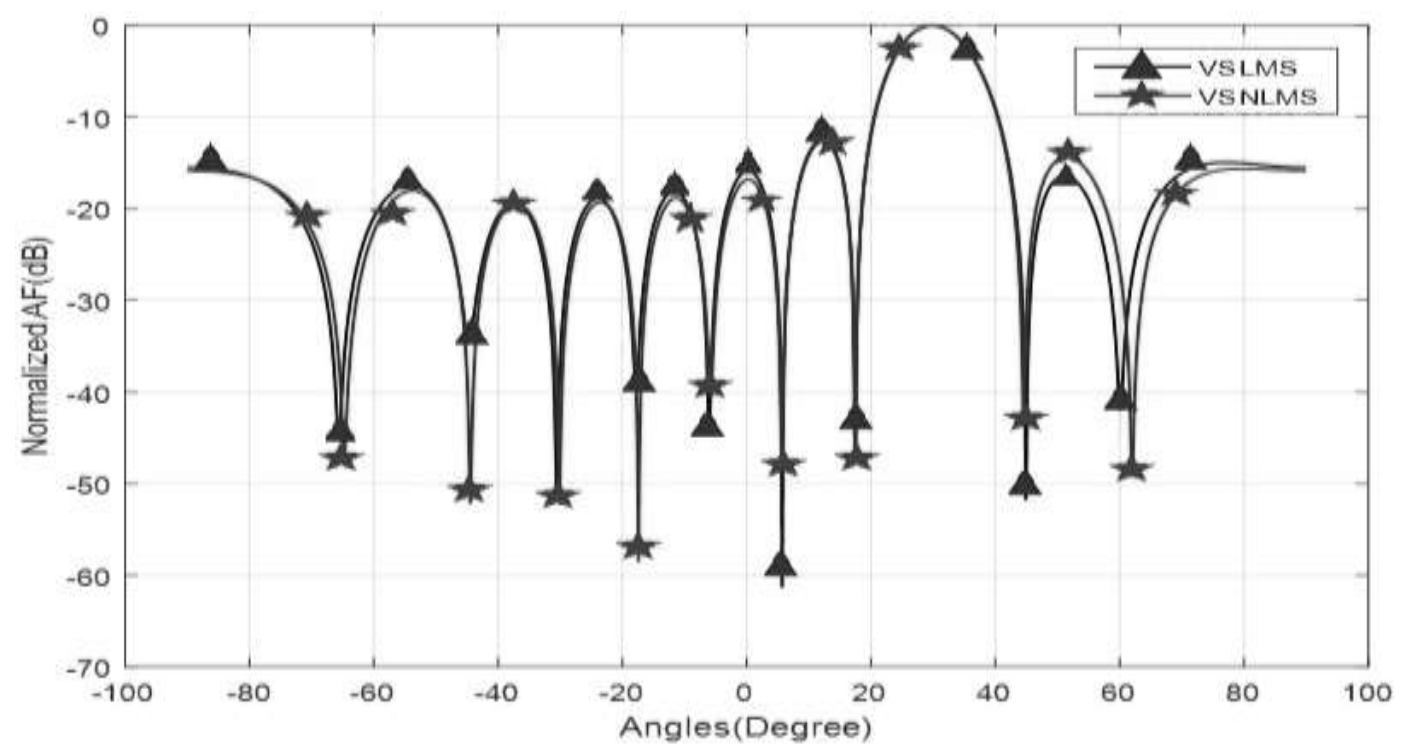

Figure 10: Normalized Array Factor for Antenna Element $\mathrm{N}=10$ at Beam Direction $=30^{\circ}$, Null Direction $=60^{\circ}$ and $\mu=0.01$ 
The normalized array factors obtained by using VSLMS and VSNLMS algorithms for antenna element of 10 and main beam direction at $15^{\circ}$, null at $0^{\circ}$ and for step-size $(\mu)$ values $0.04,0.03,0.02 \& 0.01$ respectively are plotted in figure 11 , figure 12 , figure 13 and figure 14 respectively.

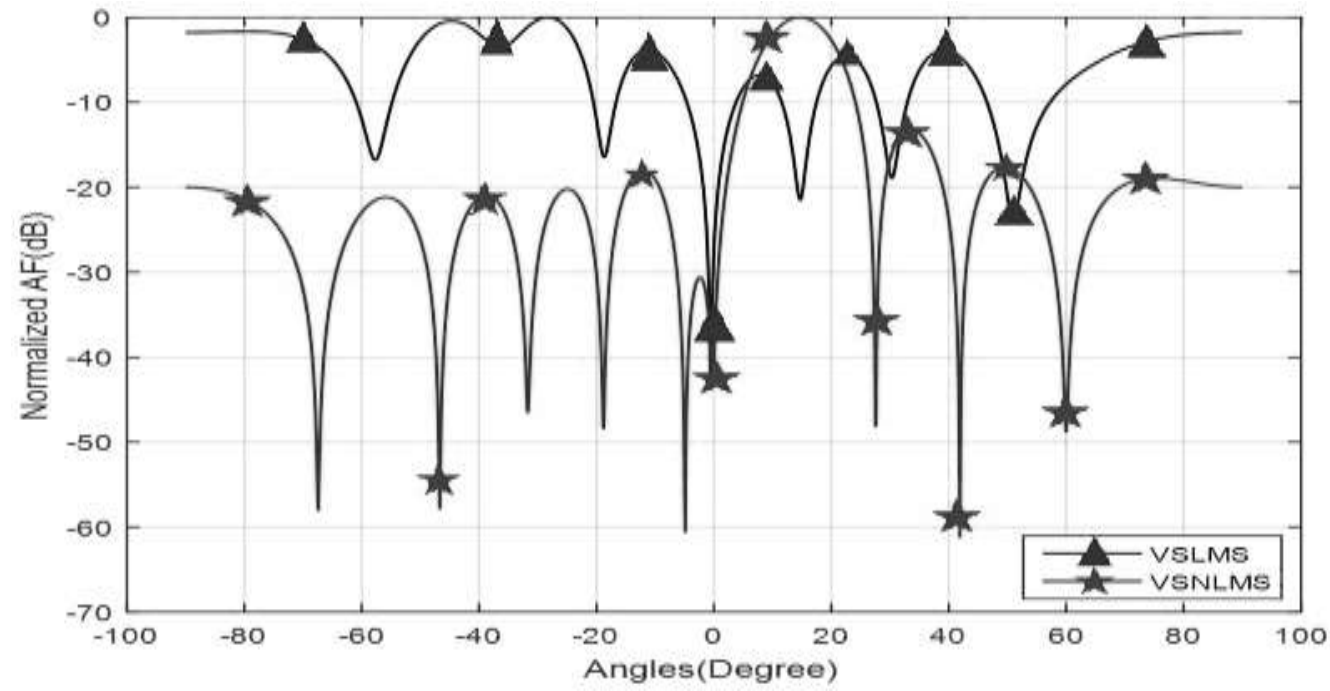

Figure 11: Normalized Array Factor for Antenna Element $N=10$ at Beam Direction $=15^{\circ}$, Null Direction $=0^{\circ}$ and $\mu=0.04$

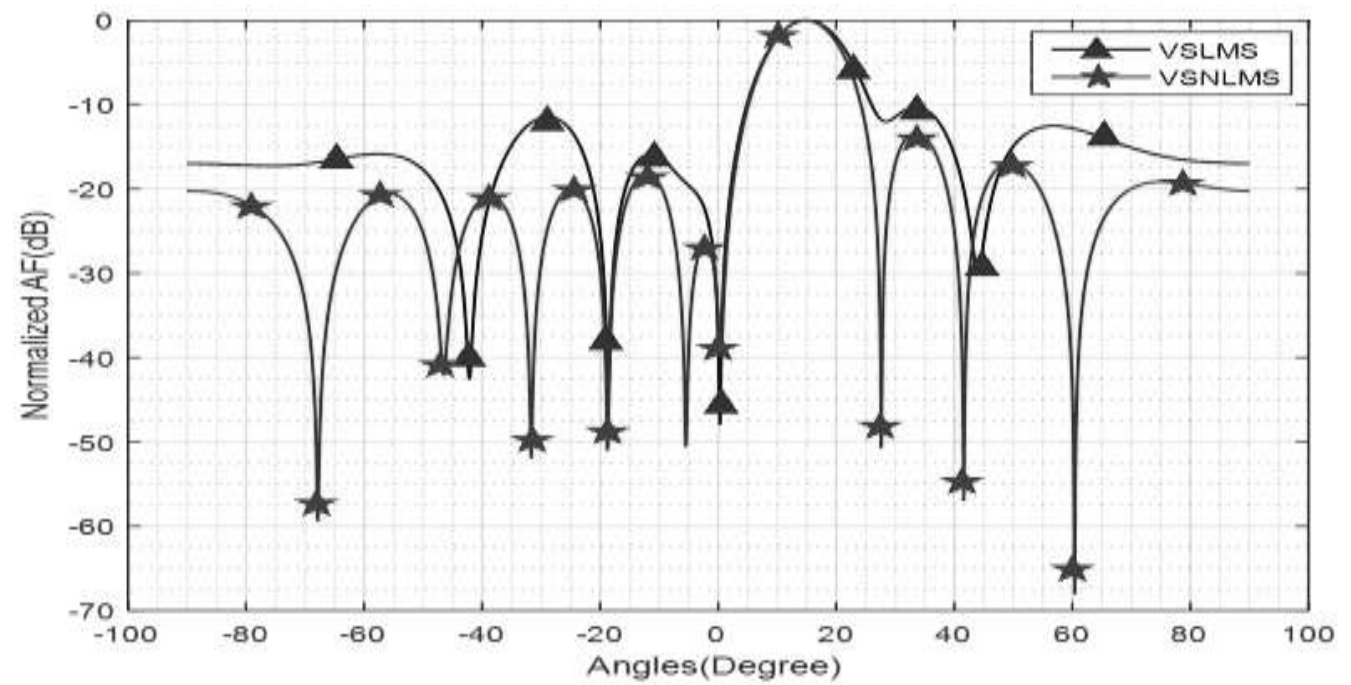

Figure 12: Normalized Array Factor for Antenna Element $N=10$ at Beam Direction $=15^{\circ}$, Null Direction $=0^{\circ}$ and $\mu=0.03$

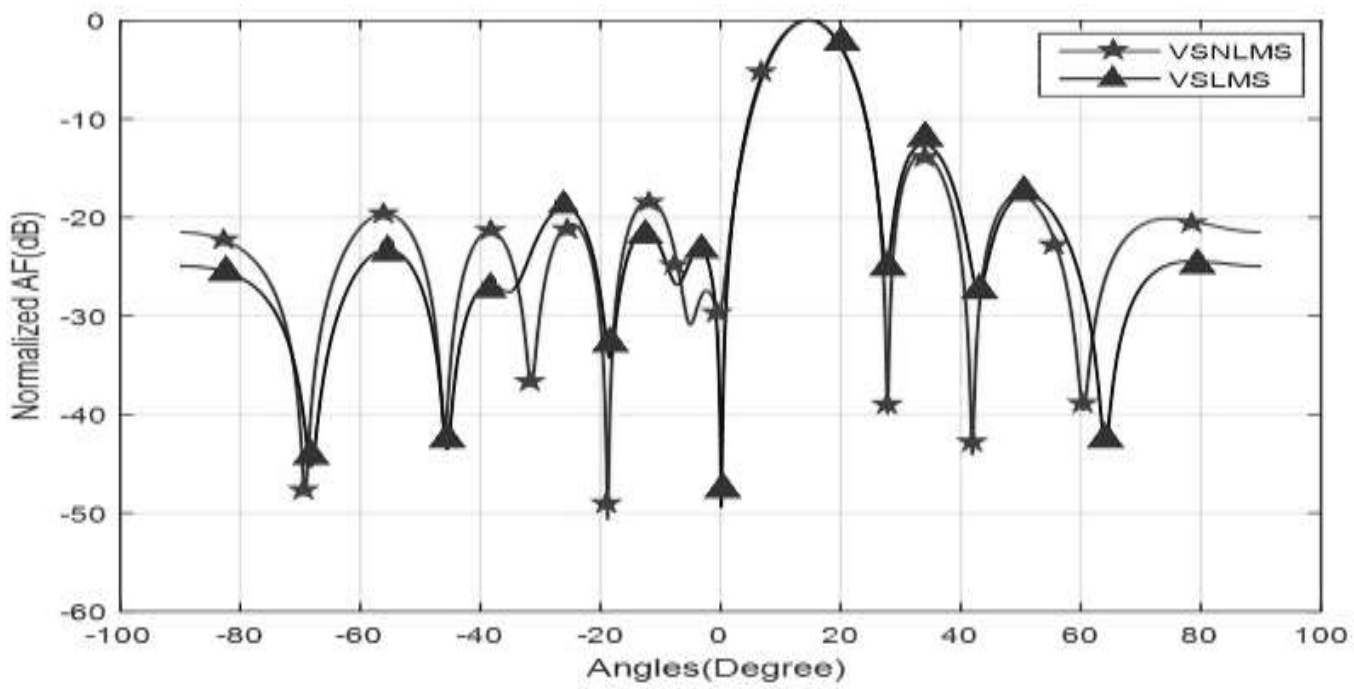

Figure 13: Normalized Array Factor for Antenna Element $N=10$ at Beam Direction $=15^{\circ}$, Null Direction $=0^{\circ}$ and $\mu=0.02$ 


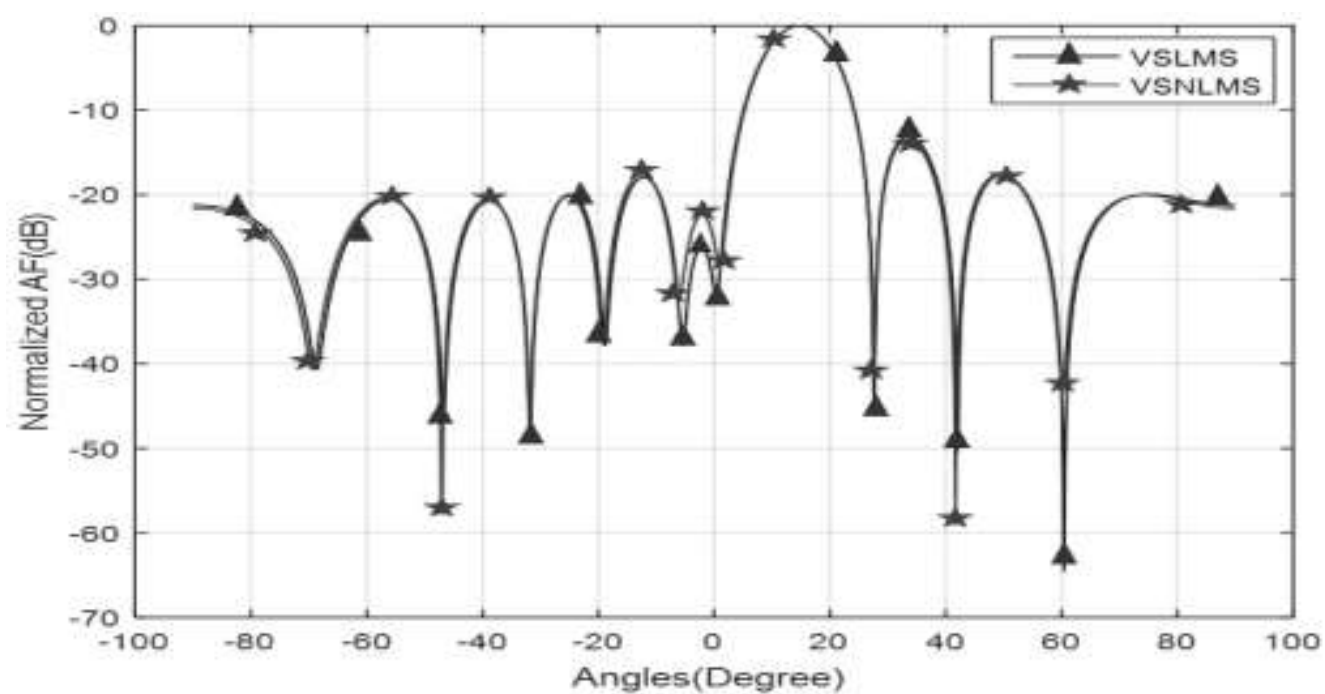

Figure 14: Normalized Array Factor for Antenna Element $N=10$ at Beam Direction $=15^{\circ}$, Null Direction $=0^{\circ}$ and $\mu=0.01$

In figure 7 and figure 11, VSLMS algorithm does not work properly for step-size value of 0.04 as it is found that LMS algorithm does not work for higher values of step-size parameter [13] and so the variable step-size LMS algorithm. The performances of VSLMS and VSNLMS algorithms for main beam direction, null depth at desired null direction and maximum side lobe level (SLLmax) are compared in Table 2.

Table 2: Performances of Beamforming Algorithms for Antenna Element N=10

\begin{tabular}{|c|c|c|c|c|c|c|}
\hline$\mu$ & $\begin{array}{c}\text { Beam \& } \\
\text { null } \\
\text { directions }\end{array}$ & Algorithms & $\begin{array}{l}\text { Simulated } \\
\text { beam } \\
\text { direction } \\
\text { (Deg) }\end{array}$ & $\begin{array}{l}\text { Null depth at } \\
\text { desired null } \\
\text { direction } \\
\text { (dB) }\end{array}$ & $\begin{array}{c}\text { Beamwidth } \\
\text { (Deg) }\end{array}$ & $\begin{array}{l}\text { SLL }_{\text {max }} \\
\text { (dB) }\end{array}$ \\
\hline \multirow{4}{*}{0.04} & \multirow{2}{*}{$15^{\circ}, 0^{\circ}$} & VSLMS & $\begin{array}{l}\text { SLL is comparable } \\
\text { with main beam }\end{array}$ & -36.0 & $\begin{array}{c}\text { Precisely cannot } \\
\text { be determined }\end{array}$ & $\begin{array}{l}\text { SLL is comparable } \\
\text { with main beam }\end{array}$ \\
\hline & & VSNLMS & $15.0^{\circ}$ & -42.0 & $26.5^{\circ}$ & -13.1 \\
\hline & \multirow{2}{*}{$30^{\circ}, 60^{\circ}$} & VSLMS & $\begin{array}{l}\text { SLL is comparable } \\
\text { with main beam }\end{array}$ & -35.5 & $\begin{array}{c}\text { Precisely cannot } \\
\text { be determined }\end{array}$ & $\begin{array}{l}\text { SLL is comparable } \\
\text { with main beam }\end{array}$ \\
\hline & & VSNLMS & $30.0^{\circ}$ & -39.5 & $27.5^{\circ}$ & -16.0 \\
\hline \multirow{4}{*}{0.03} & \multirow{2}{*}{$15^{\circ}, 0^{\circ}$} & VSLMS & $15.0^{\circ}$ & -46.0 & $27.6^{\circ}$ & -10.3 \\
\hline & & VSNLMS & $15.0^{\circ}$ & -40.0 & $27.5^{\circ}$ & -13.4 \\
\hline & \multirow{2}{*}{$30^{\circ}, 60^{\circ}$} & VSLMS & $30.0^{\circ}$ & -27.5 & $25.0^{\circ}$ & -10.0 \\
\hline & & VSNLMS & $30.0^{\circ}$ & -51.5 & $27.2^{\circ}$ & -13.0 \\
\hline \multirow{4}{*}{0.02} & \multirow{2}{*}{$15^{\circ}, 0^{\circ}$} & VSLMS & $15.0^{\circ}$ & -49.5 & $27.5^{\circ}$ & -12.6 \\
\hline & & VSNLMS & $15.0^{\circ}$ & -30.0 & $27.6^{\circ}$ & -13.6 \\
\hline & \multirow{2}{*}{$30^{\circ}, 60^{\circ}$} & VSLMS & $30.0^{\circ}$ & -26.3 & $28.5^{\circ}$ & -13.6 \\
\hline & & VSNLMS & $30.0^{\circ}$ & -37.8 & $29.0^{\circ}$ & -15.7 \\
\hline \multirow{4}{*}{0.01} & \multirow{2}{*}{$15^{\circ}, 0^{\circ}$} & VSLMS & $15.0^{\circ}$ & -32.0 & $26.5^{\circ}$ & -13.1 \\
\hline & & VSNLMS & $15.0^{\circ}$ & -27.5 & $25.5^{\circ}$ & -13.6 \\
\hline & \multirow{2}{*}{$30^{\circ}, 60^{\circ}$} & VSLMS & $30.0^{\circ}$ & -41.5 & $26.7^{\circ}$ & -12.4 \\
\hline & & VSNLMS & $30.0^{\circ}$ & -30.0 & $27.1^{\circ}$ & -12.7 \\
\hline
\end{tabular}

From Table 1 and Table 2, it is seen that for main beam directions at $15^{\circ}$ and null direction at $0^{\circ}$, as the number of antenna elements in the array increases, beamwidth decreases because array gain increases with number of elements.

Mean square error for VSLMS \& VSNLMS algorithms are plotted in Figure 15 for linear array of antenna element 8 and for main beam direction at $30^{\circ} \&$ null direction at $60^{\circ}$. Number of iterations is 200 . 


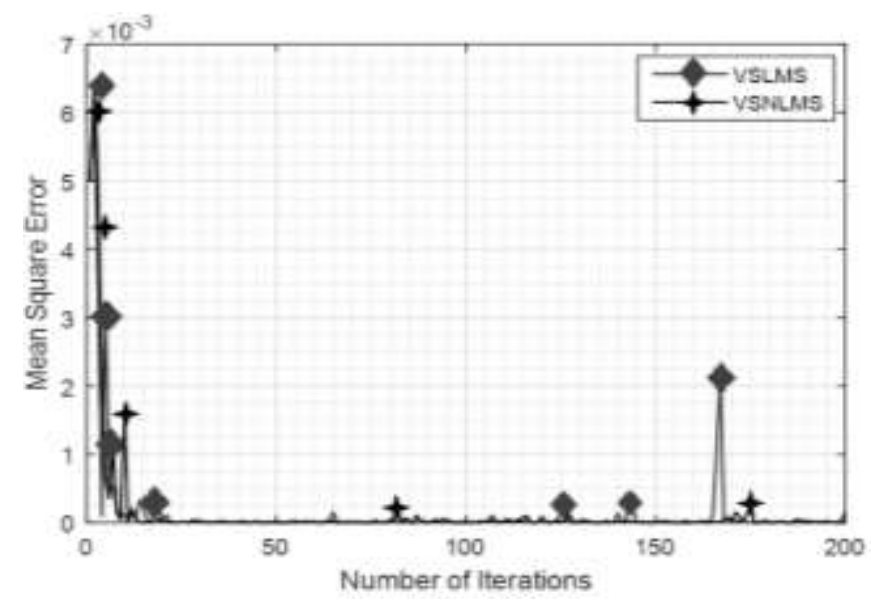

Figure 15: Mean Square Error for $\mathrm{N}=8$ with Beam at $30^{\circ}$ and Null at $60^{\circ}$

Mean square error is less and convergence is better for VSNLMS than VSLMS algorithm.

\section{DISCUSSIONS AND CONCLUSIONS}

Adaptive beamforming algorithms are simulated in MATLAB successfully and the performances of VSLMS and VSNLMS algorithms are studied on the basis of reduction of maximum side lobe level. In all the cases desired main beam directions and null directions are almost achieved. From Table $1 \&$ Table 2, it is observed that the reduction of maximum side lobe level of more than $2.5 \mathrm{~dB}$ is achieved using VSNLMS algorithm for a linear array of antenna element 8 and 10, while in the case of VSLMS algorithm, a reduced side lobe level of more $2 \mathrm{~dB}$ is achieved in comparison with LMS algorithm [8]. VSLMS algorithm does not work for higher values of step size parameter while VSNLMS algorithm works well and gives better performance. For most of the cases, VSNLMS algorithm produces deepest null and lower side lobe level. The mean square error is less and convergence is better in case of VSNLMS algorithm. So, overall the performances of VSNLMS algorithm is found better than VSLMS algorithm for reduction of maximum side lobe level which causes interference to other users. Future scope of this work may be to study the performances of the planar and circular array of smart antennas using these beamforming algorithms.

\section{REFERENCES}

[1] T. K. Sarkar, M. C. Wicks, M. Salazar-Palma, Robert J. Bonneau, Smart Antennas, Wiley-IEEE Press, 2003.

[2] F. Gross, Smart Antenna for Wireless Communication, McGraw-Hill, 2005.

[3] L. C. Gadara, "Application of antenna arrays to mobile communications, Part II: beam-forming and direction-ofarrival considerations", Proceedings of the IEEE, vol. 85, no. 8, pp. 1195-1245, Aug. 1997.

[4] A. Senapati, and J. S. Roy, "Beam-forming and beam-shaping in smart antenna-A comparative study between least mean square and recursive least square algorithms", International Journal of Microwave and Optical Technology (IJMOT), vol. 10, no. 4, pp. 232-239, July 2015.

[5] K. Ghatak, A. Senapati, and J. S. Roy, "Investigations on adaptive beamforming for linear and planar smart antenna arrays using sample matrix inversion algorithm”, Intl. Journal of Comp. Applications, vol. 117, pp. 47-50, 2015.

[6] J. A. Srar, K. S. Chung, and A. Mansour, "Adaptive array beamforming using a combined LMS-LMS algorithm", IEEE Trans. on Antennas and Propagation, vol. 58, no. 11, pp. 3545-3557, Nov. 2010.

[7] A. Senapati, and J. S. Roy, "Adaptive beam formation in smart antenna using Tchebyscheff distribution and variants of least mean square algorithm", Microwave Review, vol. 22, no. 1, pp. 11-16, Aug. 2016.

[8] M.Bairagya, A. Senapati, and J. S. Roy,"Performance of LMS and Variable Step-size LMS algorithms in adaptive smart antenna for mobile communication", International Conference on Modelling, Computing and Technological Innovations (ICMCTI), UIT Burdwan, pp.205-211, 2017.

[9] S. Haykin, Adaptive Filter Theory, Prentice Hall, 2002.

[10] Y-S. Lau, Z. M. Hussain, and R. J. Harris, "A weight vector LMS algorithm for adaptive beamforming”, IEEE TENCON Conference, New Jersey, pp.494-498, 2004.

[11]M. Rupp, "the behavior of LMS \& NLMS algorithms in the presence of spherically invariant process", IEEE Transactions on Signal Processing, vol. 41, no. 3, pp. 1149-1160, 1993.

[12] C. A. Balanis, Antenna Theory - Analysis and Design, 3rd Ed., Wiley, 2005.

[13] A. Senapati, K. Ghatak and J. S. Roy, "A comparative study of adaptive beamforming techniques in smart antenna using LMS algorithm and its variants", International Conference on Computational Intelligence \& Networks (CINE2015), IEEE Xplore, Bhubaneswar, pp. 58-62, January 2015. 